\title{
In reply: Observational study of prolonged times to tracheal extubation
}

\author{
Ariana K. Tabing, MD, MBA (10 · Jesse M. Ehrenfeld, MD, MPH • \\ Jonathan P. Wanderer, MD, MPhil
}

Received: 9 September 2015/Accepted: 15 September 2015/Published online: 22 September 2015

(C) Canadian Anesthesiologists' Society 2015

\section{To the Editor,}

We thank Dexter $e t$ al. for their interest in our work and appreciate the two points that they raised in their correspondence. ${ }^{1}$ In regard to their question on prolonged extubation, we reanalyzed our data using 28 min or greater as a cut-off time for the classification of prolonged extubation. We found that 291 cases $(2.73 \%)$ had prolonged extubation before the interventions, and 416 cases $(3.05 \%)$ had prolonged extubation after the interventions. Chi square analysis showed no significant change in prolonged extubations using this definition $(P=$ 0.14 ), consistent with results from our original analysis.

In regard to the cost of sevoflurane, we performed a careful reanalysis of our average cost of medications per

A. K. Tabing, MD, MBA ( $\square)$

Vanderbilt University School of Medicine, Nashville, TN, USA

e-mail: Ariana.tabing@gmail.com

J. M. Ehrenfeld, MD, MPH · J. P. Wanderer, MD, MPhil Department of Anesthesiology, Vanderbilt University, Nashville, TN, USA

J. M. Ehrenfeld, MD, MPH · J. P. Wanderer, MD, MPhil Department of Biomedical Informatics, Vanderbilt University, Nashville, TN, USA

J. M. Ehrenfeld, MD, MPH

Department of Health Policy and Surgery, Vanderbilt University, Nashville, TN, USA case and agree that our average cost of sevoflurane per case did appear low. Reanalysis exposed an error in our pricing data; specifically, we provided our sevoflurane price in dollars per ounce rather than in dollars per bottle. When corrected, we found our mean cost of sevoflurane to be $\$ 4.65$ per case instead of $\$ 0.63$ per case during the study period. Our median cost of sevoflurane was $\$ 3.85$ per case.

Using this corrected cost data for sevoflurane, we recalculated the mean cost of anesthetic drugs per case before and after the interventions and found that our total drug costs increased slightly. Nevertheless, there was minimal difference in the calculated cost savings between the two interventions. Before the interventions, the mean (SD) cost of anesthetic drugs per case was \$34.07 (\$55.51). Following the interventions, the mean (SD) cost of anesthetic drugs per case was \$23.25 (\$29.98). This maintains a significant decrease in the cost of anesthetic drugs per case of $\$ 10.82(P<0.001)$. Our original calculated mean cost was $\$ 10.95$, representing a difference of $1.2 \%$.

Taking these changes into account, we then recalculated the top-down projected annual cost savings by extrapolating the $\$ 10.82$ per case to the 32,823 cases performed per year. This yielded a total cost savings of $\$ 355,243.17$ rather than $\$ 359,411.85$ we originally reported. When considering the changes in case usage of the three drugs and their substituted agents, the bottom-up projected annual cost savings showed a recalculated cost savings of $\$ 230,426.91$ rather than $\$ 233,857.57$ (revised Table 5). Though our conclusions and the statistical significance of our outcomes remain unchanged, we appreciate the opportunity that this correspondence provided to identify and correct these cost calculations for sevoflurane. 
TABLE 5 Comparison of cost and usage changes with commonly substituted agents

\begin{tabular}{lllll}
\hline & Cost per case & Change in use & $\begin{array}{l}\text { Estimated cost difference } \\
\text { per year }(32,823 \text { cases })\end{array}$ & Total cost difference \\
\hline Remifentanil & $\$ 68.14$ & $-5.91 \%$ & $-\$ 132,180.64$ & $-\$ 127,075.81$ \\
Sufentanil & $\$ 9.66$ & $+1.61 \%$ & $\$ 5,104.83$ & $-\$ 109,182.43$ \\
Desflurane & $\$ 13.20$ & $-25.2 \%$ & $\mathbf{\$ 3 , 9 6 8 . 3 0}$ & $\mathbf{- \$ 1 0 3 , 3 5 1 . 1 0}$ \\
Sevoflurane & $\$ \mathbf{4 . 6 5}$ & $+2.6 \%$ & $\mathbf{\$ 1 , 8 6 3 . 0 3}$ & $\mathbf{- \$ 2 3 0 , 4 2 6 . 9 1}$ \\
Isoflurane & $\$ 0.33$ & $+17.2 \%$ & & \\
\end{tabular}

\section{(Revisions in bold)}

\section{Conflicts of interest None declared.}

Funding Dr. Wanderer is supported by the Foundation for Anesthesia Education and Research (FAER) and the Anesthesia Quality Institute (AQI) Mentored Research Training Grant in Health Services Research (MRTG-HSR).

\section{Reference}

1. Dexter F, Epstein RH. Observational study of prolonged times to tracheal extubation. Can J Anesth 2016; 63: this issue. DOI: 10. 1007/s12630-015-0496-7. 